\title{
The Multicell Processing Capacity of the Cellular MIMO Uplink Channel under Correlated Fading
}

\author{
Symeon Chatzinotas, Muhammad Ali Imran, Reza Hoshyar \\ Centre for Communication Systems Research \\ University of Surrey, United Kingdom, GU2 7XH \\ Email: \{S.Chatzinotas, M.Imran, R.Hoshyar\}@ surrey.ac.uk
}

\begin{abstract}
In the information-theoretic literature, it has been widely shown that multicell processing is able to provide high capacity gains in the context of cellular systems and that the per-cell sum-rate capacity of multicell processing systems grows linearly with the number of Base Station (BS) receive antennas. However, the majority of results in this area has been produced assuming that the fading coefficients of the MIMO subchannels are totally uncorrelated. In this direction, this paper investigates the ergodic per-cell sum-rate capacity of the MIMO Cellular Multiple-Access Channel under correlated fading and multicell processing. More specifically, the current channel model considers Rayleigh fading, uniformly distributed User Terminals (UTs) over a planar cellular system and power-law path loss. Furthermore, both BSs and Uts are equipped with correlated multiple antennas, which are modelled according to the Kronecker model. The per-cell sum-rate capacity closed form is derived using a Free Probability approach and numerical results are produced by varying the cell density of the system, as well as the level of correlation.
\end{abstract}

\section{INTRODUCTION}

In the information-theoretic literature, the majority of multicell processing capacity results has been produced based on the simplifying assumption that the fading coefficients of the MIMO subchannels are totally uncorrelated. In general, correlation in MIMO point-to-point channels can be caused by inadequate antenna separation and/or poor local scattering [1]. More specifically, if the components of an antenna array are separated by a distance less than half of the communication wavelength, then the fading coefficients appear correlated. Furthermore, if the number of local scatterers is insufficient, then the regularities between the multiple paths can lead to correlated fading. In a typical macrocellular scenario, the inadequate antenna separation mainly affects the UTs due to their size limitations, whereas poor local scattering affects the BSs due to their elevated position. Assuming that there are no keyhole effects, the channel matrix can be written as $\mathbf{H}=\mathbf{R}_{R}^{1 / 2} \mathbf{G} \mathbf{R}_{T}^{1 / 2}$ where $\mathbf{G}$ is a Gaussian matrix. The matrices $\mathbf{R}_{R}$ and $\mathbf{R}_{T}$, a.k.a. the receive and transmit correlation matrix, depend on the angle spread, the antenna beamwidth and the antenna spacing at the receive and the transmit end respectively. This channel matrix represents the Kronecker correlation model, since the covariance of the vectorized channel matrix can be written as the Kronecker product of the receive and transmit correlation matrix, namely $\operatorname{cov}(\operatorname{vec}(\mathbf{H}))=\mathbf{R}_{R} \otimes \mathbf{R}_{T}$. According to the Kronecker correlation model, the transmit correlation is independent of the receive antenna and vice versa. Furthermore, the correlation between two subchannels equals to the product of the corresponding transmit and receive correlation. From a physical point-of-view, the Kronecker model appears when the antennas are arranged in regular arrays and the correlation vanishes fast with distance. It has been well established in the literature [2] that the Kronecker model can be transformed into a separable correlation model, while studying the eigenvalue distribution of $\mathbf{H H}^{\dagger}$. More specifically, if $\mathbf{R}_{R}=\mathbf{U D}_{R} \mathbf{U}^{\dagger}$ and $\mathbf{R}_{T}=\mathbf{V} \mathbf{D}_{R} \mathbf{V}^{\dagger}$ are the eigenvalue decompositions of the receive and transmit correlation matrices respectively, then the eigenvalue distribution of $\mathbf{H} \mathbf{H}^{\dagger}=\mathbf{R}_{R}^{1 / 2} \mathbf{G} \mathbf{R}_{T} \mathbf{G}^{\dagger} \mathbf{R}_{R}^{1 / 2}$ is equivalent to the one of $\mathbf{D}_{R}^{1 / 2} \mathbf{G D}_{T} \mathbf{G}^{\dagger} \mathbf{D}_{R}^{1 / 2}$. In this direction, the equivalent MIMO channel matrix can be written as $\mathbf{H} \asymp \mathbf{D}_{R}^{1 / 2} \mathbf{G D}_{T}^{1 / 2}$ where $\asymp$ denotes equivalence of the eigenvalue distribution of $\mathbf{H H}^{\dagger}$. The performance of multiantenna channels was originally investigated in [3] and it was shown that the capacity grows linearly with $\min \left(n_{r}, n_{t}\right)$, where $n_{r}$ and $n_{t}$ are the number of receive and transmit antennas respectively. However, the correlated fading amongst the multiple antennas can compromise the capacity performance w.r.t. the independent fading case. In the literature, the majority of the approaches consider the asymptotic setting where correlation affects both transmit and receive end and the numbers of both transmit and receive antennas grow large together while preserving a fixed ratio. Although the asymptotic analysis comprises an approximation for matrices of finite dimensions, it is often employed in order to isolate the effect of specific physical parameters and to produce analytical closed forms. This setting is particularly suitable for studying the uplink channel of multicell processing cellular systems, since the ratio of transmit and receive antennas is a constant proportional to the per-cell number of UTs $K$. In [2], the capacity of the Kronecker MIMO channel is expressed as a fixed-point equation based on the Steltjes' transform of the asymptotic eigenvalue distribution (a.e.d.) of $\mathbf{H H}^{\dagger}$. In the same direction, authors in [4] study the capacity of the Kronecker MIMO channel based on the principles of Random Matrix Theory [5]. The derivation results in a fixed-point equation including functionals of the $S I N R$ and $M M S E$. In [6] and [7], the expectation and the variance of the capacity are evaluated using closed forms based on the solution of $2 \times 2$ equation systems. In [8], the principles 
of majorization theory are applied in order to show that the average mutual information is a Schur-concave function with respect to the ordered eigenvalue vector of the correlation matrix. In addition, the Kronecker MIMO channel for Toeplitz correlation matrices is analyzed in [9] based on the concept of linear spectral statistics. Finally, in [10] the performance of Kronecker correlated MIMO channels is studied using the replica method, which originates in theoretical physics. It should be noted that the aforementioned results focus on the point-to-point correlated MIMO channel. Subsequently, we describe the channel matrix characteristics of a cellular multiple-access channel which forms the basis of modelling the uplink channel of multicell processing cellular systems. It should be noted that when the transmitter has no Channel State Information (CSI), the capacity of MIMO channel with uniform power allocation coincides with the capacity of a MIMO Multiple Access Channel (MAC). However, the channel model of the cellular channel is affected by an additional variance profile which is dictated by the considered path loss model. The rest of this section describes the evolution of the pathloss variance profile in the context of multicell processing literature. The description starts with single-antenna cellular systems and concludes with the extension of the channel model for multiple-antennas at both transmit and receive ends.

Initially, Wyner [11] introduced the concept of interference factor $a$, which quantifies the amount of intercell interference with the first interfering tier. Subsequently, Somekh and Shamai [12] introduced the independent identically distributed (i.i.d.) fading coefficients. Multiple tiers of interference were introduced by Letzepis [13], which has considered variable interference factors depending on the distance from the interfering tier and the power-law path loss. Finally, Chatzinotas et al [14] have alleviated the assumption of collocated UTs by introducing user distribution. In the latter model, by assuming power-law path loss, flat fading and uniformly distributed users, the received signal at cell $n=1 \ldots N$, at time index $t$, is given by:

$$
y^{n}[t]=\sum_{m=1}^{N} \sum_{k=1}^{K} \varsigma_{k}^{n m} g_{k}^{n m}[t] x_{k}^{m}[t]+z^{n}[t],
$$

where $x_{k}^{m}[t]$ is the $t$ th complex channel symbol transmitted by the $k$ th UT of the $m$ th cell and $\left\{g_{k}^{n m}\right\}$ are independent, strictly stationary and ergodic complex random processes, which represent the flat fading processes experienced in the transmission path between the $n$th BS and the $k$ th UT in the $m$ th cell. The fading coefficients are assumed to have unit power, i.e. $\mathbb{E}\left[\left\|g_{k}^{n m}[t]\right\|^{2}\right]=1$ for all $(n, m, k)$ and all UTs are subject to an average power constraint, i.e. $\mathbb{E}\left[\left\|x_{k}^{m}[t]\right\|^{2}\right] \leq P$ for all $(m, k)$. The interference factors $\varsigma_{k}^{n m}$ in the transmission path between the $m$ th BS and the $k$ th UT in the $n$th cell are calculated according to the "modified" power-law path loss model [13]: $\varsigma_{k}^{n m}=\left(1+d_{k}^{n m}\right)^{-\eta / 2}$, where $\eta$ is the path loss exponent. Dropping the time index $t$, the aforementioned model can be compactly expressed as a vector memoryless channel of the form: $\mathbf{y}=\mathbf{H x}+\mathbf{z}$. The channel matrix $\mathbf{H}$ can be written as

$$
\mathbf{H}=\boldsymbol{\Sigma} \odot \mathbf{G},
$$

where $\boldsymbol{\Sigma}$ is a $N \times K N$ deterministic matrix and $\mathbf{G} \sim$ $\mathcal{C N}\left(\mathbf{0}, \mathbf{I}_{N}\right)$ is a Gaussian $N \times K N$ matrix, comprising the corresponding Rayleigh fading coefficients. The entries of the $\Sigma$ matrix are defined by the variance profile function

$$
\varsigma(u, v)=(1+d(u, v))^{-\frac{\eta}{2}},
$$

where $u \in[0,1]$ and $v \in[0, K]$ are the normalized indexes for the BSs and the UTs respectively and $d(u, v)$ is the normalized distance between BS $u$ and user $v$. In the case of multiple UT and/or BS antennas ( $n_{U T}$ and $n_{B S}$ respectively), the channel matrix $\mathbf{H}$ can be written as, $\mathbf{H}=\boldsymbol{\Sigma}_{M} \odot \mathbf{G}_{M}$, where $\mathbf{G}_{M} \sim$ $\mathcal{C N}\left(\mathbf{0}, \mathbf{I}_{N n_{B S}}\right)$ is a complex Gaussian $N n_{B S} \times K N n_{U T}$ matrix, comprising the Rayleigh fading coefficients between the $K N n_{U T}$ transmit and the $N n_{B S}$ receive antennas. Similarly, $\boldsymbol{\Sigma}_{M}$ is a $N n_{B S} \times K N n_{U T}$ deterministic matrix, comprising the path loss coefficients between the $K N n_{U T}$ transmit and the $N n_{B S}$ receive antennas. Since the multiple antennas of each UT / BS are collocated, $\boldsymbol{\Sigma}_{M}$ can be written as a block matrix based on the variance profile matrix $\boldsymbol{\Sigma}$ of Equation (2) $\boldsymbol{\Sigma}_{M}=\boldsymbol{\Sigma} \otimes \mathbf{J}$, where $\mathbf{J}$ is a $n_{B S} \times n_{U T}$ matrix of ones.

\section{Channel Model \& Analysis}

Assume that the $K$ UTs are uniformly distributed in each cell of a planar cellular system comprising $N$ base stations and that each BS and each UT are equipped with $n_{B S}$ and $n_{U T}$ antennas respectively. Under conditions of correlated flat fading, the received signal at cell $n$, at time index $t$, will be given by:

$$
\begin{aligned}
\mathbf{y}^{n}[t] & =\sum_{m=1}^{N} \sum_{k=1}^{K} \varsigma_{k}^{n m}\left(\mathbf{R}_{\mathbf{R} k}^{n m}\right)^{\frac{1}{2}} \mathbf{G}_{k}^{n m}[t]\left(\mathbf{R}_{\mathbf{T}}^{n m}\right)^{\frac{1}{2}} \mathbf{x}_{k}^{m}[t] \\
& +\mathbf{z}^{n}[t]
\end{aligned}
$$

where $\mathbf{x}_{k}^{m}[t]$ is the $t$ th complex channel symbol vector $n_{U T} \times 1$ transmitted by the $k$ th UT of the $m$ th cell and $\left\{\mathbf{G}_{k}^{n m}\right\}$ is a $n_{U T} \times n_{U T}$ random matrix with independent, strictly stationary and ergodic complex circularly symmetric (c.c.s.) i.i.d. elements. $\mathbf{R}_{\mathbf{T}}{ }^{n m}$ and $\mathbf{R}_{\mathbf{R}}{ }_{k}^{n m}$ are deterministic transmit and receive correlation matrices of dimensions $n_{U T} \times n_{U T}$ and $n_{B S} \times n_{B S}$ respectively. In this context, the following normalizations are considered in order to ensure that the correlation matrices do not affect the path loss gain of the BSUT links: $\operatorname{tr}\left(\mathbf{R}_{\mathbf{T} k}^{n m}\right)=n_{U T}$ and $\operatorname{tr}\left(\mathbf{R}_{\mathbf{R}}^{n m}\right)=n_{B S}$ for all $(n, m, k)$. The matrix product $\left(\mathbf{R}_{\mathbf{R}_{k} m}^{n m}\right)^{1 / 2} \mathbf{G}_{k}^{n m}[t]\left(\mathbf{R}_{\mathbf{T}}^{n m}\right)^{1 / 2}$ represents the multiple-antenna correlated flat fading processes experienced in the transmission path between the $n_{B S}$ receive antennas of the $n$th BS and the $n_{U T}$ transmit antennas of the $k$ th UT in the $m$ th cell. The fading coefficients are assumed to have unit power, i.e. $\mathbb{E}\left[\mathbf{G}_{k}^{n m}[t] \mathbf{G}_{k}^{n m}[t]^{\dagger}\right]=\mathbf{I}$ for all $(n, m, k)$ and all UTs are subject to a power constraint $P$, i.e. $\mathbb{E}\left[\boldsymbol{x}_{k}^{m}[t] \boldsymbol{x}_{k}^{m}[t]^{\dagger}\right] \preceq \frac{P}{n_{U T}} \mathbf{I}_{n_{U T}}$ for all $(m, k)$. It should be noted that the UTs are assumed to be totally ignorant of the channel state information (CSI). In case the UTs had perfect of even 
statistical CSI, input optimization strategies could be used to maximize the ergodic capacity. The variance coefficients $\varsigma_{k}^{n m}$ in the transmission path between the $m$ th $\mathrm{BS}$ and the $k$ th UT in the $n$th cell are calculated according to the "modified" power-law path loss model. Dropping the time index $t$, the aforementioned model can be compactly expressed as a vector memoryless channel of the form $\mathbf{Y}=\mathbf{H X}+\mathbf{Z}$, where the vec-

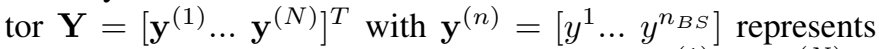
received signals by the BSs, the vector $\mathbf{X}=\left[\mathbf{x}_{(1)}^{(1)} \ldots \mathbf{x}_{(K)}^{(N)}\right]^{T}$

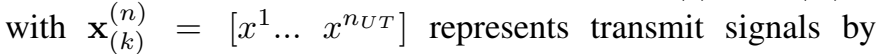
all the UTs of the cellular system and the components of

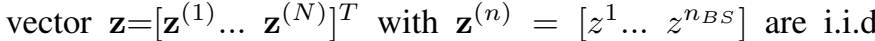
c.c.s. random variables representing AWGN with $\mathbb{E}\left[\mathbf{z}^{n}\right]=$ $\mathbf{0}, \mathbb{E}\left[\mathbf{z}^{n}[t] \mathbf{z}^{n}[t]^{\dagger}\right]=\sigma^{2} \mathbf{I}$. Based on the a.e.d. equivalence, the channel matrix can be rewritten as:

$$
\begin{aligned}
& \mathbf{H}=\boldsymbol{\Sigma}_{\mathbf{M}} \odot\left(\left(\mathbf{I}_{N} \otimes \mathbf{R}_{\mathbf{R}}^{\frac{1}{2}}\right) \mathbf{G}_{\mathbf{M}}\left(\mathbf{I}_{K N} \otimes \mathbf{R}_{\mathbf{T}}^{\frac{1}{2}}\right)\right) \\
& \asymp \boldsymbol{\Sigma}_{\mathbf{M}} \odot\left(\tilde{\mathbf{D}}_{\mathbf{R}}^{\frac{1}{2}} \mathbf{G}_{\mathbf{M}} \tilde{\mathbf{D}}_{\mathbf{T}}^{\frac{1}{2}}\right)=\boldsymbol{\Sigma}_{\mathbf{M}} \odot\left(\tilde{\mathbf{d}}_{\mathbf{R}}^{\dagger} \tilde{\mathbf{d}}_{\mathbf{T}}\right)^{\frac{1}{2}} \odot \mathbf{G}_{\mathbf{M}}
\end{aligned}
$$

where $\tilde{\mathbf{D}}_{\mathbf{R}}$ and $\tilde{\mathbf{D}}_{\mathbf{T}}$ are the diagonal eigenvalue matrices of $\mathbf{I}_{N \times N} \otimes \mathbf{R}_{\mathbf{R}}$ and $\mathbf{I}_{N \times N} \otimes \mathbf{R}_{\mathbf{T}}$ respectively and $\tilde{\mathbf{d}}_{\mathbf{R}}$ and $\tilde{\mathbf{d}}_{\mathbf{T}}$ are row vectors containing the diagonal elements of $\tilde{\mathbf{D}}_{\mathbf{R}}$ and $\tilde{\mathbf{D}}_{\mathbf{T}}$ respectively. As it can be seen, the MIMO correlation model has been transformed into an uncorrelated model with a variance profile $\Omega=\Sigma_{\mathbf{M}} \odot\left(\tilde{\mathbf{d}}_{\mathbf{R}}^{\dagger} \tilde{\mathbf{d}}_{\mathbf{T}}\right)^{1 / 2}$, which is neither row regular [5, Def. 2.10] nor separable. The rest of this section describes a free probability approach which can be utilized to find the a.e.d. in the case of single-side and double-side Kronecker correlation. Free probability has been also used in [15] to investigate the case of point-to-point MIMO channels correlated on a single side according to the exponential model.

According to [5], the asymptotic sum-rate capacity $C_{\text {opt }}$ for the uncorrelated model assuming a very large number of cells, is given by

$$
\begin{aligned}
& C_{\mathrm{opt}}=\lim _{N \rightarrow \infty} \frac{1}{N} \mathcal{I}(\mathbf{x} ; \mathbf{y} \mid \mathbf{H}) \\
& =\lim _{N \rightarrow \infty} \mathbb{E}\left[\frac{1}{N} \sum_{i=1}^{N n_{B S}} \log \left(1+\frac{\tilde{\gamma}}{K n_{U T}} \lambda_{i}\left(\frac{1}{N} \mathbf{H H}^{\dagger}\right)\right)\right] \\
& =n_{B S} \int_{0}^{\infty} \log \left(1+\frac{\tilde{\gamma}}{K n_{U T}} x\right) d \mathrm{~F}_{\frac{1}{N} \mathbf{H H}^{\dagger}}(x) \\
& =n_{B S} \mathcal{V}_{\frac{1}{N}} \mathbf{H H}^{\dagger}\left(\frac{\tilde{\gamma}}{K n_{U T}}\right)=n_{B S} K n_{U T} \mathcal{V}_{\frac{1}{N}} \mathbf{H}^{\dagger} \mathbf{H}\left(\frac{\tilde{\gamma}}{K n_{U T}}\right)
\end{aligned}
$$

where $\tilde{\gamma}=K N \gamma$ and $\gamma=P / \sigma^{2}$ are the system- and UT-transmit power normalized by the receiver noise power respectively, $\lambda_{i}(\mathbf{X})$ denotes the eigenvalues of matrix $\mathbf{X}$ and $\mathcal{V}_{\mathbf{X}}(y)$ is the Shannon transform [5] of a random square Hermitian matrix $\mathbf{X}$, whose a.e.d. has a cumulative function denoted by $\mathrm{F}_{\mathbf{X}}(x)$. For a Gaussian matrix $\mathbf{G}$, the a.e.d. of $\frac{1}{N} \mathbf{G}^{\dagger} \mathbf{G}$ converges almost surely (a.s.) to the nonrandom a.e.d. of the Marčenko-Pastur law

$$
\mathcal{V}_{\frac{1}{N} \mathbf{G}^{\dagger} \mathbf{G}}(y) \stackrel{a . s .}{\longrightarrow} \mathcal{V}_{\mathrm{MP}}(y, K) .
$$

However, considering the uncorrelated MIMO cellular channel it applies that $\mathbf{H}=\mathbf{\Sigma}_{\mathbf{M}} \odot \mathbf{G}_{\mathbf{M}}$. In this case, the a.e.d. of $\frac{1}{N} \mathbf{H} \mathbf{H}^{\dagger}$ is derived based on the analysis in [13] and using tools from the discipline of Free Probability. In this direction, $\frac{1}{N} \mathbf{H}^{\dagger} \mathbf{H}$ can be written as the sum of $K N n_{U T} \times K N n_{U T}$ unit rank matrices, i.e.

$$
\frac{1}{N} \mathbf{H}^{\dagger} \mathbf{H}=\sum_{i=1}^{N n_{B S}} \mathbf{h}_{i}^{\dagger} \mathbf{h}_{i}
$$

where $\mathbf{h}_{i} \sim \mathcal{C N}\left(\mathbf{0}, \mathbf{V}_{i}\right)$ denotes the $i$ th $1 \times K N n_{U T}$ row vector of $\frac{1}{\sqrt{N}} \mathbf{H}$, since the term $\frac{1}{N}$ has been incorporated in the unit rank matrices. The covariance matrix equals $\mathbf{V}_{i}=$ $\frac{1}{N}\left(\operatorname{diag}\left(\boldsymbol{\sigma}_{i}\right)\right)^{2}$, where $\operatorname{diag}\left(\boldsymbol{\sigma}_{i}\right)$ stands for a diagonal matrix with the elements of vector $\boldsymbol{\sigma}_{i}$ across the diagonal with $\boldsymbol{\sigma}_{i}$ being the $i$ th row of $\boldsymbol{\Sigma}_{\mathbf{M}}$. The unit-rank matrices $\mathbf{W}_{i}=\mathbf{h}_{i}^{\dagger} \mathbf{h}_{i}$ constitute complex singular Wishart matrices with one degree of freedom and their density according to [16] is

$$
\begin{aligned}
& f_{\mathbf{V}_{i}}\left(\mathbf{W}_{i}\right)=B_{\mathbf{V}_{i}}^{-1} \operatorname{det}\left(\mathbf{W}_{i}\right)^{1-K n_{U T} N} e^{-\operatorname{tr}\left(\mathbf{V}_{i}^{-1} \mathbf{W}_{i}\right)} \\
& B_{\mathbf{V}_{i}}=\pi^{K n_{U T} N-1} \operatorname{det}\left(\mathbf{V}_{i}\right) .
\end{aligned}
$$

It can be easily seen that if $\mathbf{V}_{i}=\mathbf{I}$, the matrices would be unitarily invariant and therefore asymptotically free [5, Example 2.46]. Although in our case $\mathbf{V}_{i}=\frac{1}{N}\left(\operatorname{diag}\left(\boldsymbol{\sigma}_{i}\right)\right)^{2}$, we assume that the asymptotic freeness still holds. Similar approximations have already been investigated in an information-theoretic context providing useful analytical insights and accurate numerical results [17], [18]. In this context, the R-transform of each unit rank matrix [5, Example 2.28] is given by

$$
\mathcal{R}_{\mathbf{h}_{\mathbf{i}}^{\dagger}{ }^{\dagger} \mathbf{h}_{\mathbf{i}}}(w)=\frac{1}{K N n_{U T}} \frac{\left\|\mathbf{h}_{i}\right\|^{2}}{1-w\left\|\mathbf{h}_{i}\right\|^{2}} .
$$

and the asymptotic R-transform of $\frac{1}{N} \mathbf{H}^{\dagger} \mathbf{H}$ is equal to the sum of the R-transforms of all the unit rank matrices [5, Th. 2.64]

$$
\begin{aligned}
\lim _{N \rightarrow \infty} \mathcal{R}_{\frac{1}{N} \mathbf{H}^{\dagger} \mathbf{H}}(w) & \simeq \lim _{N \rightarrow \infty} \sum_{i=1}^{N n_{B S}} \mathcal{R}_{\mathbf{h}_{\mathbf{i}}^{\dagger} \mathbf{h}_{\mathbf{i}}}(w) \\
& =\lim _{N \rightarrow \infty} \frac{1}{K N n_{U T}} \sum_{i=1}^{N n_{B S}} \frac{\left\|\mathbf{h}_{i}\right\|^{2}}{1-w\left\|\mathbf{h}_{i}\right\|^{2}} .
\end{aligned}
$$

Since the variance profile function of Equation (3) defines rectangular block-circulant matrix with $1 \times K$ blocks which is symmetric about $u=K v$, the channel matrix $\mathbf{H}$ is asymptotically row-regular and thus the asymptotic norm of $\mathbf{h}_{i}$ converges to a deterministic constant for every BS, i.e $\forall i$

$$
\lim _{N \rightarrow \infty}\left\|\mathbf{h}_{i}\right\|^{2}=\lim _{N \rightarrow \infty} \frac{1}{N} \sum_{j=1}^{K N n_{U T}} \varsigma_{i j}^{2}=\int_{0}^{K n_{U T}} \varsigma^{2}(u, v) d v
$$

where $\varsigma_{i j}$ is the $(i, j)$ th element of the $\boldsymbol{\Sigma}_{\mathbf{M}}$ matrix. In addition, based on the row-regularity it can be seen that

$$
n_{B S} \int_{0}^{K n_{U T}} \varsigma^{2}(u, v) d v=\int_{0}^{n_{B S}} \int_{0}^{K n_{U T}} \varsigma^{2}(u, v) d u d v .
$$


Therefore, the R-transform can be simplified to [5, Th. 2.31, Ex. 2.26]

$$
\begin{aligned}
& \lim _{N \rightarrow \infty} \mathcal{R}_{\frac{1}{N} \mathbf{H}^{\dagger} \mathbf{H}}(w) \\
& \simeq \frac{1}{K n_{U T}} \int_{0}^{n_{B S}} \frac{\int_{0}^{K n_{U T}} \varsigma^{2}(u, v) d v}{1-w \int_{0}^{K n_{U T}} \varsigma^{2}(u, v) d v} d u \\
& =\frac{1}{K n_{U T}} \frac{\int_{0}^{n_{B S}} \int_{0}^{K n_{U T}} \varsigma^{2}(u, v) d u d v}{n_{B S}-w \int_{0}^{n_{B S}} \int_{0}^{K n_{U T}} \varsigma^{2}(u, v) d u d v} \\
& =q\left(\boldsymbol{\Sigma}_{\mathbf{M}}\right) \frac{1}{1-\frac{K n_{U T}}{n_{B S}} w q\left(\boldsymbol{\Sigma}_{\mathbf{M}}\right)}=\mathcal{R}_{q\left(\boldsymbol{\Sigma}_{\mathbf{M}}\right) \frac{1}{N} \mathbf{G}_{\mathbf{M}}^{\dagger} \mathbf{G}_{\mathbf{M}}(w)}
\end{aligned}
$$

where $q\left(\boldsymbol{\Sigma}_{M}\right) \triangleq\left\|\boldsymbol{\Sigma}_{M}\right\|^{2} /\left(K N^{2} n_{U T} n_{B S}\right)$ is the Frobenius norm of the $\boldsymbol{\Sigma}_{\mathbf{M}}$ matrix $\left\|\boldsymbol{\Sigma}_{\mathbf{M}}\right\| \triangleq \sqrt{\operatorname{tr}\left\{\boldsymbol{\Sigma}_{\mathbf{M}}{ }^{\dagger} \boldsymbol{\Sigma}_{\mathbf{M}}\right\}}$ normalized with the matrix dimensions and

$$
\left\|\boldsymbol{\Sigma}_{M}\right\|^{2}=\operatorname{tr}\left\{(\boldsymbol{\Sigma} \otimes \mathbf{J})^{\dagger}(\boldsymbol{\Sigma} \otimes \mathbf{J})\right\}=\|\boldsymbol{\Sigma}\|^{2} n_{U T} n_{B S} .
$$

Thus, it can be seen that $q\left(\boldsymbol{\Sigma}_{M}\right)=q(\boldsymbol{\Sigma})=\|\boldsymbol{\Sigma}\|^{2} /\left(K N^{2}\right)$. In the asymptotic case, $q(\boldsymbol{\Sigma})$ is given by $\lim _{N \rightarrow \infty} q(\boldsymbol{\Sigma})=$ $\frac{1}{K} \int_{0}^{K} \varsigma^{2}(u, v) d v$. The a.e.d. of $\frac{1}{N} \mathbf{H}^{\dagger} \mathbf{H}$ follows a scaled version of the Marčenko-Pastur law and hence the Shannon transform of the a.e.d. of $\frac{1}{N} \mathbf{H}^{\dagger} \mathbf{H}$ can be approximated by

$$
\mathcal{V}_{\frac{1}{N} \mathbf{H}^{\dagger} \mathbf{H}}\left(\frac{\tilde{\gamma}}{K n_{U T}}\right) \simeq \mathcal{V}_{\mathrm{MP}}\left(q(\boldsymbol{\Sigma}) \frac{\tilde{\gamma}}{K n_{U T}}, K n_{U T}\right)
$$

As a result, the per-cell capacity is given by

$$
C_{\mathrm{opt}} \simeq n_{B S} K n_{U T} \mathcal{V}_{\mathrm{MP}}\left(q(\boldsymbol{\Sigma}) \frac{\tilde{\gamma}}{K n_{U T}}, K n_{U T}\right) .
$$

1) UT-side correlation: In this case, we assume that $\mathbf{R}_{\mathbf{T}}$ has the same eigenvalues for all UTs. The following analysis can be easily generalized to encompass the case of different transmit correlation matrix at each UT, but we keep this assumption to simplify the notations. Assuming that there is no receive correlation at the $\mathrm{BS}$ side i.e $\mathbf{R}_{\mathbf{R}}=\mathbf{I}$, the channel matrix of Equation (5) can be rewritten as follows:

$$
\frac{1}{\sqrt{N}} \mathbf{H}=\left(\mathbf{W}\left(\mathbf{I}_{K N} \otimes \mathbf{R}_{\mathbf{T}}^{\frac{1}{2}}\right)\right) \asymp\left(\mathbf{W}\left(\mathbf{I}_{K N} \otimes \mathbf{D}_{\mathbf{T}^{\frac{1}{2}}}\right)\right)
$$

where $\mathbf{W}=\frac{1}{\sqrt{N}} \boldsymbol{\Sigma}_{\mathbf{M}} \odot \mathbf{G}_{\mathbf{M}}$ and therefore

$$
\begin{aligned}
& \frac{1}{N} \mathbf{H}^{\dagger} \mathbf{H}=\sum_{i=1}^{N n_{B S}} \mathbf{h}_{i}^{\dagger} \mathbf{h}_{i} \\
& \asymp \sum_{i=1}^{N n_{B S}}\left(\mathbf{I}_{K N} \otimes \mathbf{D}_{\mathbf{T}}^{\frac{1}{2}}\right) \mathbf{w}_{i}^{\dagger} \mathbf{w}_{i}\left(\mathbf{I}_{K N} \otimes \mathbf{D}_{\mathbf{T}}{ }^{\frac{1}{2}}\right) \\
& =\sum_{i=1}^{N n_{B S}}\left(\left(\mathbf{1}_{K N} \otimes \lambda_{\mathbf{T}}^{\frac{1}{2}}\right) \odot \mathbf{w}_{i}\right)^{\dagger}\left(\left(\mathbf{1}_{K N} \otimes \lambda_{\mathbf{T}}^{\frac{1}{2}}\right) \odot \mathbf{w}_{i}\right)
\end{aligned}
$$

where $\mathbf{w}_{i}$ denotes the $i$ th $1 \times K N n_{U T}$ row vector of $\mathbf{W}$, $\mathbf{1}_{K N}$ is a $1 \times K N$ row vector of ones and $\lambda_{\mathbf{T}}$ is a row vector containing the eigenvalues of $\mathbf{R}_{\mathbf{T}}$. Hence, the R-transform can be written as

$$
\lim _{N \rightarrow \infty} R_{\frac{1}{N} \mathbf{H}^{\dagger} \mathbf{H}}(w)=\mathcal{R}_{q(\Omega) \frac{1}{N} \mathbf{G}_{\mathbf{M}}^{\dagger} \mathbf{G}_{\mathbf{M}}}(\omega)
$$

$$
\begin{gathered}
\text { where } q(\boldsymbol{\Omega})=\frac{\left\|\mathbf{h}_{i}\right\|^{2}}{K N n_{U T}}=\frac{\left\|\left(\mathbf{1}_{K N} \otimes \lambda_{\mathbf{T}}^{\frac{1}{2}}\right) \mathbf{w}_{i}\right\|^{2}}{K N n_{U T}} \\
=\frac{1}{n_{U T}} \sum_{j=1}^{n_{U T}} \lambda_{\mathbf{T}}(j) \cdot \frac{1}{K} \int_{0}^{K} \varsigma^{2}(u, v) d v=q(\boldsymbol{\Sigma})
\end{gathered}
$$

As a result, the per-cell capacity is given by (17) which coincides with the case of uncorrelated multiple antennas. Therefore, we can conclude for large values of $K\left(K \gg n_{U T}\right)$ transmit correlation has no effect on the system's performance. This ascertainment is expected, since the capacity scaling is dictated by the rank of the channel matrix $\mathbf{H}$, which depends on the number of BS antennas in a cellular scenario.

2) BS-side correlation: In this case, we assume that $\mathbf{R}_{\mathbf{R}}$ has the same eigenvalues for all BSs. The following analysis can be easily generalized to encompass the case of receive different correlation matrix at each BS. Assuming that there is no transmit correlation at the UT side i.e. $\mathbf{R}_{\mathbf{T}}=\mathbf{I}$, the channel matrix of Equation (5) can be rewritten as follows:

$$
\begin{gathered}
\frac{1}{\sqrt{N}} \mathbf{H}=\left(\left(\mathbf{I}_{N} \otimes \mathbf{R}_{\mathbf{R}}^{\frac{1}{2}}\right) \mathbf{W}\right) \asymp\left(\left(\mathbf{I}_{N} \otimes \mathbf{D}_{\mathbf{R}}{ }^{\frac{1}{2}}\right) \mathbf{W}\right) \\
\text { and } \frac{1}{N} \mathbf{H}^{\dagger} \mathbf{H}=\frac{1}{N} \sum_{i=1}^{N} \mathbf{H}_{i}^{\dagger} \mathbf{H}_{i} \\
=\sum_{i=1}^{N} \mathbf{W}_{i}^{\dagger} \mathbf{D}_{\mathbf{R}} \mathbf{W}_{i}=\sum_{j=1}^{n_{B S}} \lambda_{\mathbf{R}}(j) \sum_{i=1}^{N} \mathbf{w}_{i}^{\dagger} \mathbf{w}_{i}
\end{gathered}
$$

where $\mathbf{H}_{i}$ and $\mathbf{W}_{i}$ are submatrices of $\mathbf{H}$ and $\mathbf{W}$ respectively with dimensions $n_{B S} \times K N n_{U T}$ and $\lambda_{\mathbf{R}}$ is a row vector containing the eigenvalues of $\mathbf{R}_{\mathbf{R}}$. Based on the previous analysis, the a.e.d. of $\mathbf{A}=\sum_{i=1}^{N} \mathbf{w}_{i}^{\dagger} \mathbf{w}_{i}$ follows a scaled version of the Marčenko-Pastur law. Hence, the R-transform of $\mathbf{A}$ can be written as

$$
\mathcal{R}_{\mathbf{A}}(w) \asymp \mathcal{R}_{q(\boldsymbol{\Sigma}) \frac{1}{N}} \tilde{\mathbf{G}}^{\dagger} \tilde{\mathbf{G}}(w)=\frac{q(\boldsymbol{\Sigma})}{1-K n_{U T} w q(\boldsymbol{\Sigma})}
$$

where $\tilde{\mathbf{G}} \sim \mathcal{C N}\left(\mathbf{0}, \mathbf{I}_{\mathbf{N}}\right)$ is a $N \times K N n_{U T}$ Gaussian matrix The R-transform of $\frac{1}{N} \mathbf{H}^{\dagger} \mathbf{H}$ is based on [5, Th. 2.31,2.64]

$$
\mathcal{R}_{\mathbf{H}^{\dagger} \mathbf{H}}(w)=\sum_{j=1}^{n_{B S}} \lambda_{\mathbf{R}}(j) \mathcal{R}_{\mathbf{A}}\left(\lambda_{\mathbf{R}}(j) w\right)
$$

The asymptotic eigenvalue pdf (AEPDF) of $\frac{1}{N} \mathbf{H}^{\dagger} \mathbf{H}$ is obtained by determining the imaginary part of the Cauchy transform $\mathcal{G}$ for real arguments $f^{\infty}(x)=$ $\lim _{y \rightarrow 0^{+}} \frac{1}{\pi} \mathfrak{I}\{\mathcal{G}(x+j y)\}$, considering that the Cauchy transform is derived from the R-transform as $\mathcal{G}^{-1}(w)=\mathcal{R}(-w)-$ $\frac{1}{w}$. However, in order to calculate the channel sum-rate capacity, the AEPDF of $\frac{1}{N} \mathbf{H} \mathbf{H}^{\dagger}$ is needed. The matrices $\frac{1}{N} \mathbf{H} \mathbf{H}^{\dagger}$ and $\frac{1}{N} \mathbf{H}^{\dagger} \mathbf{H}$ have the same non zero eigenvalues, but their sizes differ by a factor of $K n_{U T} / n_{B S}$. This implies that $f_{\mathbf{H H}^{\dagger}}^{\infty}(x)=K \frac{n_{U T}}{n_{B S}} f_{\mathbf{H}^{\dagger} \mathbf{H}}^{\infty}(x)$. Finally, the per-cell capacity is

$$
\begin{aligned}
C_{\mathrm{opt}} & =n_{B S} \int_{0}^{\infty} \log \left(1+\frac{\tilde{\gamma}}{K n_{U T}} x\right) f_{\frac{1}{N} \mathbf{H H}^{\dagger}}^{\infty}(x) d x \\
& =K n_{U T} \int_{0}^{\infty} \log \left(1+\frac{\tilde{\gamma}}{K n_{U T}} x\right) f_{\frac{1}{N} \mathbf{H}^{\dagger} \mathbf{H}}^{\infty}(x) d x .
\end{aligned}
$$




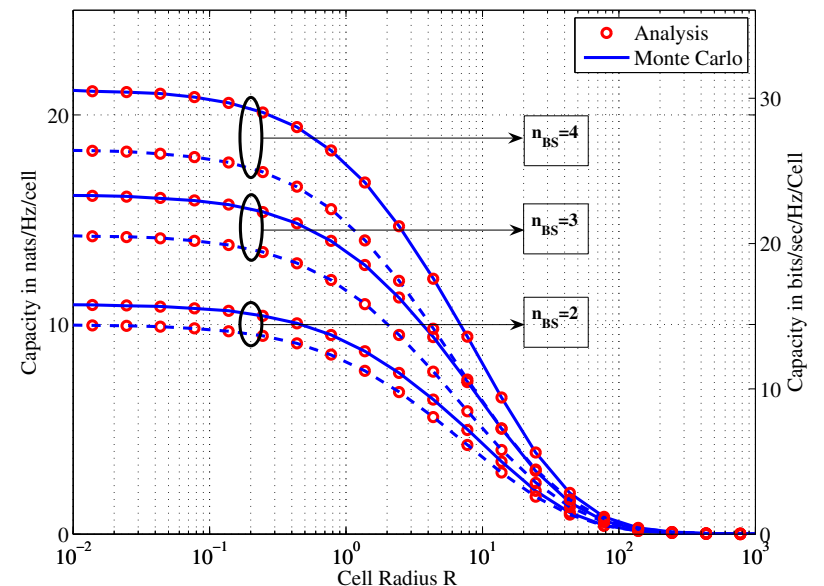

Fig. 1. Per-cell sum-rate capacity vs. the normalized cell Radius $R$ varying the number of $\mathrm{BS}$ antennas $n_{B S}$ for 2 values of receive correlation $\rho_{R}=[0,0.8]$ (solid and dashed line respectively). Parameters: $K=4, \gamma=$ $10, n_{U T}=2, \eta=2$.

3) Double-side correlation: By combining the two previous cases, it can be easily seen that the a.e.d. for the doubleside Kronecker correlation model coincides with the BS-side correlation case, since UT-side correlation has no effect on the a.e.d. of $\frac{1}{N} \mathbf{H H}^{\dagger}$.

\section{NumericAl ReSUlTS \& CONCLUSION}

The analytical results have been verified by running Monte Carlo simulations. More specifically, for each system instance the channel matrix is constructed by randomly generating correlated fading coefficients according to the exponential model [15] with $\rho_{R}$ and $\rho_{T}$ being the receive and transmit correlation coefficient respectively. Subsequently, the path-loss variance profile is constructed by randomly placing the UTs according to the considered distribution in the coverage area of each cell and by calculating the variance profile coefficients using Equation (3). In the context of the mathematical analysis, the UTs are assumed to be positioned on a uniform planar grid. The simulated sum-rate capacity is calculated using [3]

$$
C_{\text {opt }}(\gamma)=\frac{1}{N} \mathbb{E}\left[\operatorname{logdet}\left(\mathbf{I}_{N n_{B S}}+\frac{\gamma}{n_{U T}} \mathbf{H H}^{\dagger}\right)\right] \text {. }
$$

Figure 1 depicts the per-cell sum-rate capacity vs. the normalized cell radius $R$ varying the number of BS antennas $n_{B S}$ for 2 values of receive correlation $\rho_{R}=[0,0.8]$ in a planar cellular system with uniformly distributed UTs. As it can be seen, the receive correlation decreases the degrees of freedom due to the multiple receive antennas and therefore compromises the capacity performance of the system. It also becomes clear that correlation does not affect the linear multiplexing gain with the number of receive antennas $n_{B S}$. Nevertheless, correlation results in a decreased growth rate with respect to the uncorrelated case.

\section{ACKNOWLEDGMENT}

The work reported in this paper has formed part of the "Fundamental Limits to Wireless Network Capacity" Elec- tive Research Programme of the Virtual Centre of Excellence in Mobile \& Personal Communications, Mobile VCE, www.mobilevce.com. This research has been funded by the following Industrial Companies who are Members of Mobile VCE - BBC, BT, Huawei, Nokia, Nokia Siemens Networks, Nortel, Vodafone. Fully detailed technical reports on this research are available to staff from these Industrial Members of Mobile VCE. The authors would like to thank Prof. G. Caire and Prof. D. Tse for the useful discussions.

\section{REFERENCES}

[1] D.-S. Shiu, G. Foschini, and M. G. J. Kahn, "Fading correlation and its effect on the capacity of multielement antenna systems," IEEE Trans. Commun., vol. 48, no. 3, pp. 502-513, Mar 2000.

[2] C.-N. Chuah, D. Tse, J. Kahn, and R. Valenzuela, "Capacity scaling in MIMO wireless systems under correlated fading," IEEE Trans. Inform. Theory, vol. 48, no. 3, pp. 637-650, Mar 2002.

[3] I. E. Telatar, "Capacity of multi-antenna Gaussian channels," European Transactions on Telecommunications, vol. 10, no. 6, pp. 585-595, Nov 1999.

[4] A. Tulino, A. Lozano, and S. Verdu, "Impact of antenna correlation on the capacity of multiantenna channels," IEEE Trans. Inform. Theory, vol. 51, no. 7, pp. 2491-2509, Jul 2005.

[5] A. M. Tulino and S. Verdu, "Random matrix theory and wireless communications," Commun. Inf. Theory, vol. 1, no. 1, pp. 1-182, 2004.

[6] A. Sengupta and P. Mitra, "Capacity of multivariate channels with multiplicative noise: Random matrix techniques and large-n expansions (2)," Journal of Statistical Physics, vol. 125, pp. 1223-1242(20), Dec 2006.

[7] W. Hachem, O. Khorunzhiy, P. Loubaton, J. Najim, and L. Pastur, "A new approach for mutual information analysis of large dimensional multi-antenna channels," IEEE Trans. Inform. Theory, vol. 54, no. 9, pp. 3987-4004, Sep 2008.

[8] E. Jorswieck and H. Boche, "Performance analysis of MIMO systems in spatially correlated fading using matrix-monotone functions," IEICE Transactions, vol. 89-A, no. 5, pp. 1454-1472, 2006.

[9] H. Shin, M. Win, and M. Chiani, "Asymptotic statistics of mutual information for doubly correlated MIMO channels," IEEE Trans. Wireless Commun., vol. 7, no. 2, pp. 562-573, Feb 2008.

[10] A. Moustakas and S. Simon, "On the outage capacity of correlated multiple-path MIMO channels," IEEE Trans. Inform. Theory, vol. 53, no. 11, pp. 3887-3903, Nov 2007.

[11] A. Wyner, "Shannon-theoretic approach to a Gaussian cellular multipleaccess channel," IEEE Trans. Inform. Theory, vol. 40, no. 6, pp. 17131727, Nov 1994.

[12] O. Somekh and S. Shamai, "Shannon-theoretic approach to a Gaussian cellular multiple-access channel with fading," IEEE Trans. Inform. Theory, vol. 46, no. 4, pp. 1401-1425, Jul 2000.

[13] N. Letzepis, "Gaussian cellular muptiple access channels," Ph.D. dissertation, Institute for Telecommunications Research, University of South Australia, Dec 2005.

[14] S. Chatzinotas, M. Imran, and C. Tzaras, "On the capacity of variable density cellular systems under multicell decoding," IEEE Commun. Lett., vol. 12, no. 7, Jul 2008.

[15] X. Mestre, J. Fonollosa, and A. Pages-Zamora, "Capacity of MIMO channels: asymptotic evaluation under correlated fading," IEEE J. Select. Areas Commun., vol. 21, no. 5, pp. 829-838, June 2003.

[16] T. Ratnarajah and R. Vaillancourt, "Complex random matrices and applications," Computer \& Mathematics with Applications, vol. 50, no. 3-4, pp. 399-411, Aug 2005.

[17] M. Peacock, I. Collings, and M. Honig, "Asymptotic spectral efficiency of multiuser multisignature CDMA in frequency-selective channels," IEEE Trans. Inform. Theory, vol. 52, no. 3, pp. 1113-1129, Mar 2006.

[18] W. Hachem, "Low complexity polynomial receivers for downlink CDMA," Signals, Systems and Computers, 2002. Conference Record of the Thirty-Sixth Asilomar Conference on, vol. 2, pp. 1919-1923 vol.2, Nov 2002. 